\title{
Association of Serum Nonesterified Fatty Acid and Insulin Resistance with Nonalcoholic Fatty Liver Disease-A Study from Bangladeshi Prediabetic Subjects
}

\author{
Israt Ara Hossain ${ }^{1}$, Mijanur Rahman Shah ${ }^{2}$ and Liaquat $\mathrm{Ali}^{1}{ }^{*}$ \\ ${ }^{1}$ Department of Biochemistry and Cell Biology, Bangladesh University of Health Sciences, Dhaka, Bangladesh \\ ${ }^{2}$ Department of Microbiology and Immunology, Bangabandhu Sheikh Mujib Medical University, Shahbag, Dhaka, Bangladesh
}

*Corresponding author: Liaquat Ali (MBBS, MPhil, PhD), Professor, Department of Biochemistry and Cell Biology, Bangladesh University of Health Sciences, Dhaka, Bangladesh, Tel: +88-02-9010932; +88-02-901952; Fax: +88-02-8055312; E-mail: vc@buhs.edu.bd

Received date: August 31, 2018; Accepted date: September 21, 2018; Published date: September 28, 2018

Copyright: (C) 2018 Hossain IA, et al. This is an open-access article distributed under the terms of the Creative Commons Attribution License, which permits unrestricted use, distribution, and reproduction in any medium, provided the original author and source are credited.

\begin{abstract}
Objective: Portal entry of nonesterified fatty acid (NEFA) into the hepatocytes due to insulin resistance is a leading cause of nonalcoholic fatty liver disease (NAFLD). Herein, we assessed the relationship between elevated NEFA and insulin resistance levels with NAFLD among Bangladeshi prediabetic individuals.

Methods: A total of 151 prediabetic subjects (M/F, 85/66; age in years, $45.08 \pm 9.37$; $\mathrm{BMl}$ in $\mathrm{kg} / \mathrm{m}^{2}, 25.95 \pm 4.42$; $M \pm S D$ ) after OGTT confirmation were purposively recruited in this cross-sectional study and divided into two groups according to their liver ultrasonogram: non NAFLD (47/37; $44.11 \pm 9.35 ; 25.37 \pm 4.65)$ and NAFLD (38/29; $46.30 \pm 9.33 ; 26.66 \pm 4.04$ ). Diabetes and prediabetes was diagnosed as per WHO Study Group criteria. Serum NEFA was measured by the enzymatic colorimetric method and serum insulin by ELISA technique. Insulin resistance (HOMA-IR) was calculated by homeostasis model assessment (HOMA).
\end{abstract}

Results: Compared to subjects with non NAFLD, NAFLD subjects showed significantly higher levels of fasting serum NEFA $(0.62 \pm 0.25$ vs. $0.50 \pm 0.26 \mathrm{mmol} / \mathrm{L}, \mathrm{p}=0.010)$ and HOMA-IR $(2.21 \pm 1.01$ vs. $1.79 \pm 0.51, p=0.001)$. On binary logistic regression analysis, NEFA (odds ratio [OR]: $6.18 ; 95 \%$ confidence interval [Cl]: 1.54 to 24.84 , $p=0.010)$ and HOMA-IR (OR=1.92; $95 \% \mathrm{Cl}: 1.09$ to $3.40, p=0.024)$ were found to be significant determinants of NAFLD after adjusting the effects of potential confounders of age, BMI, and triglyceride respectively. On multiple linear regression analysis, NEFA showed significant negative association with insulin sensitivity $(\beta=-1.849, p=0.049)$ after adjusting the effects of other pertinent covariates of BMI, glycemic and postprandial serum insulin respectively.

Conclusion: Increased levels of serum NEFA is an independent determinant of NAFLD in Bangladeshi prediabetic population and it seems that the elevation is associated with reduced insulin sensitivity during this disorder.

Keywords: Nonesterified fatty acid; Insulin resistance; Nonalcoholic fatty liver disease; Prediabetes; Bangladesh

\section{Introduction}

Nonalcoholic fatty liver disease (NAFLD) has been recognized as a clinicopahological condition caused by excessive accumulation of fat within the hepatocytes. It is characterized by simple steatosis caused by excessive fat deposition and can be progressed into nonalcoholic steatohepatitis (NASH), where oxidative stress, inflammation and hepatocellular damage with or without fibrosis and cirrhosis are common. The disease affects $10-30 \%$ of the general population in western countries and 5-8\% in Asia [1,2]. The prevalence of NAFLD in obese and type 2 diabetic individual is estimated to be approximately $70-90 \%$ with the consequences of liver related morbidity and mortality [2]. Recent studies revealed that NAFLD is associated not only with hepatic disorders but also with extra-hepatic chronic complications such as cardiovascular diseases (CVDs), type 2 diabetes mellitus (T2DM), obesity, dyslipidemia, hypertension, and insulin resistance and constitutes a new cluster of the metabolic syndrome (MetS) [3].
During fatty liver, loss of insulin sensitivity increases the lipotoxicity within the hepatocytes which ultimately enhances the lipogenesis by reesterification of fatty acids with the glycerol moiety. Although the mechanisms underlying liver disease progression remain unclear, insulin resistance, obesity related ectopic fat following inflammation and lipotoxicity play a key role, along with possible genetic, dietary and lifestyle factors [4].

Another important factor is nonesterified fatty acids (NEFAs), secreted from adipose tissue via lipolysis of stored triacylglycerol (TAG) from its glycerol backbone. Although the subcutaneous fat cell enhances the uptake of NEFAs a small proportion of the systemic NEFA concentrations arise within the hepatocytes from visceral fat depots [5]. After absorption of dietary fats, the mobilization of stored and dietary fats is suppressed rapidly by insulin whereas, any meal that contains carbohydrates there is increased secretion of insulin which in turn reduces the NEFA concentrations by increasing its $\beta$ oxidation within the liver mitochondria. After an overnight fast, plasma NEFA concentrations elevated rapidly which in turn declines following a meal [6]. In the fasting state, hormone sensitive adipose triglyceride 
lipase (ATGL) enhances the hydrolysis of stored TAG in the adipose tissue with the release of NEFAs and enters the circulation by binding with albumin. The entry of NEFAs within the extrahepatic tissues is facilitated by specific fatty acid transporter where it undergoes $\beta$ oxidation with the release of energy; however, an impaired insulin action enhances the production of newly made fatty acid by reesterification of the glycerol moiety and subsequent accumulation of TAG within the hepatocytes [7].

In the postprandial state, increased levels of insulin promote the mobilization of dietary fats in the form of chylomicron remnants which then incorporated into TAG within the hepatocytes but a proportion of fats always join the portal NEFA pool in a process sometimes called spillover that accounts $40-50 \%$ of the total plasma NEFA pool. Fifty nine percent of the TAG that accumulates in the liver during NAFLD is derived from circulating FFAs [8]. Accordingly, insulin resistance which is the hallmark feature of NAFLD increases peripheral lipolysis, with the availability of NEFA and subsequently causing microvascular dysfunction with the development and progression of NAFLD [9]. However, a recent study by Finucane et al. claimed that postprandial NEFA rather the fasting NEFA plays important role in whole body insulin resistance among the healthy elderly males [6]. Prior epidemiological studies revealed IR as a predominant risk factor for NAFLD where body fat distribution appears to play a more important role in the pathogenesis of NAFLD [9].

Adipose depots play a crucial role in the increased production of NEFAs via lipolysis of stored fat which eventually converted into TAG within the hepatocytes during insulin resistant state leading to the onset of NAFLD. The prevalence of NAFLD among the T2DM individuals has been increased concomitantly in Bangladesh [10]; hence, greater attention should be imposed on prediabetic screening to combat the disorder. An elevation of plasma NEFA considered important biomarker for the development of NAFLD while a number of studies confirmed a significant association of NEFA levels with insulin resistance syndrome in the different population [3,4]. However, very little studies have documented the association of NEFA levels with insulin resistance in prediabetic individuals who are NAFLD [11]. Considering the importance of adipose tissue derived factors in the development of NAFLD during the insulin resistant state which is one of the basic defects of prediabetes, the role of plasma NEFA levels during this state needs to be elucidated for the prevention and management of the disorder. In this context, the present study has been assessed to explore the association of increased serum NEFA with NAFLD and to investigate whether this association is mediated by insulin resistance among prediabetic individuals.

\section{Materials and Methods}

\section{Subjects and study design}

A cross-sectional study was carried out on 151 prediabetic subjects, after confirmation by OGTT were purposively recruited who came between November 2012 and March 2013 to check their routine health assessment in BIHS General Hospital, Dhaka, Bangladesh. Diabetes and prediabetes were diagnosed employing WHO Group Study criteria [12]. Informed written consent was taken from each participant prior to study inclusion for use of their study related information and for participation in ongoing research. Upper abdomen ultrasonogram had done of all study subjects and were divided into 84 non NAFLD and 67 NAFLD groups which then further stratified into grade $0(n=84)$, grade $1(n=53)$, grade $2(n=11)$, and grade $3(n=3)$ respectively depending on different grades of NAFLD. The study was approved by the Ethical review committee of Bangladesh Diabetic Association (BADAS). Ref no: BADAS-ERC/13/00106. All experiments were performed in accordance with relevant guidelines and regulations of the Institutes. Subjects who were alcoholic, had liver cirrhosis, viral hepatitis, hepatobiliary diseases, pancreatitis, inflammatory bowel disease, thyroid abnormalities, cancer, chronic kidney disorder, type 1 \& 2 diabetes, chronic cardiac and respiratory diseases, stroke, using lipid and glucose lowering drugs and pregnant subjects were excluded from our study. The clinical and anthropometric evaluations included measures of systolic and diastolic blood pressures (SBP and DBP), waist and hip circumference (WC and $\mathrm{HC}$ ), waist to hip ratio (WHR) and body mass index (BMI). BMI of the subjects was calculated by the formula: BMI=body weight $(\mathrm{kg}) /$ height $^{2}\left(\mathrm{~m}^{2}\right)$. The study subjects were stratified into obese and hypertensive based on WHO and WHO/ISH guidelines $[13,14]$.

\section{Biochemical analysis}

Fasting venous blood samples were collected after overnight fasting (8-14 hours) [15] between 8.00-9.00 am and immediately followed by a 75-g OGTT to assess the biochemical tests. Fasting serum glucose (FSG) and $2 \mathrm{~h}$ postprandial serum glucose (PPSG) were measured by the glucose-oxidase method and glycated haemoglobin $\mathrm{A}_{1 \mathrm{c}}\left(\mathrm{HbA}_{1 \mathrm{c}}\right)$ levels were determined by high performance liquid chromatography technique. Total cholesterol (TC), triglyceride (TG), high-density lipoprotein cholesterol (HDL-c), aspartate transaminase (AST), alanine transaminase (ALT), gamma-glutamyl transaminase (GGT) and alkaline phosphatase (ALP) levels were assessed by using a standard enzymatic method and low-density lipoprotein cholesterol (LDL-c) was calculated by Friedwald formula [16]. Serum nonesterified fatty acid (NEFA) levels were measured by enzymatic colorimetric method. The glycemic, lipidemic and liver enzyme profile were assessed by using a clinical chemistry autoanalyzer (Dimension ${ }^{\circ}$ RxL clinical chemistry system, Siemens Healthcare Diagnostics Inc. USA). $\mathrm{HbA}_{1 \mathrm{c}}$ levels were determined by using an automated system (Bio-Rad variant and TURBO, USA). The concentration of fasting serum insulin and $2 \mathrm{~h}$ postprandial serum insulin was quantified by an ELISA technique using the plate reader (Thermo scientific multiskan ${ }^{\circ}$ FC-filter-based microplate photometer, Finland). When specific performance characteristics were assessed for the measurement of NEFA, the within and between run precision for NEFA samples were $4.58 \%$ and $3.04 \%$ coefficients of variation $(\mathrm{CV})$ respectively and for insulin samples, the values were $6.3 \%$ and $4.25 \%$ respectively. Insulin secretory function (HOMA\%B) and insulin sensitivity (HOMA\%S) was calculated from fasting serum glucose and fasting serum insulin values by homeostasis model assessment (HOMA) using HOMACIGMA software. Insulin resistance was quantified by homeostasis model assessment of insulin resistance (HOMA-IR) and calculated according to the formula: HOMA-IR=Fasting serum insulin $(\mu \mathrm{IU} / \mathrm{mL})$ $\times$ fasting serum glucose $(\mathrm{mmol} / \mathrm{L}) / 22.5$ [17].

\section{NAFLD evaluation}

The ultrasonographic examination was performed in a fasting state of each participant by a skilled sonographer who was totally blind about the purpose of the study with the help of a sonography machine (Philips Ultrasound-Ay-MNT-15 TTK, HDI-4000, Netherland) with a 3.5 MHz linear transducer. The severity of echogenicity was graded as follows: normal (grade 0), mild (grade 1, slight increase in 
echogenicity), moderate (grade 2, increased echogenicity with impaired visualization of intrahepatic vessel and diaphragm), and severe (grade 3, distinct increase in hepatic echogenicity with and posterior right lobe of the liver [18]. We also calculated fatty liver index (FLI) - a surrogate indicator of liver steatosis with a cut-off value $<30$ as normal and $>30$ as moderate and $\geq 60$ as severe steatosis derived by the formula:

$F L I=\frac{\left[\begin{array}{l}e^{0.953 \times \log e(T G)+0.139 \times B M I+0.718 \times \log e(G G T)}+ \\ 0.053 \times \text { waistcircumference }-15.745)\end{array}\right]}{\left[\begin{array}{l}1+e^{0.953 \times \log e(T G)+0.139 \times B M I+0.178 \times \log e(G G T)} \\ +0.053 \times \text { waistcircumference }-15.754\end{array}\right]} \times 100[19]$

\section{Data and Statistical Analysis}

Statistical analyses were performed using the Statistical Package for Social Sciences (SPSS) software package version 15.0 for Windows (SPSS Inc., Chicago, IL, USA). The normally distributed data with continuous variables were expressed as the mean and standard deviation (SD), whereas the categorical variables were represented by frequency and percentages. Independent 2-sample t-tests were used to compare the differences between two groups. Multivariate logistic regression analysis was used to estimate the odds ratio (OR) and $95 \%$ confidence interval (CI) for the association of NAFLD with significant predictors. A multiple linear regression analysis was used to determine the effects of HOMA-IR on serum NEFA concentrations in NAFLD subjects after adjustment for covariates. Distribution of serum NEFA and HOMA-IR according to different grades of NAFLD were compared using a one way analysis of variance (ANOVA). The sample size was calculated by using the regression model for individual predictors and it depends on the desired power (l- $\alpha)$, significance level (a), the number of predictors and the expected effect sizes. Sampling weights were used by using the formula of $n \geq 50+8 \mathrm{~m}$, where $\mathrm{m}$ is the number of independent variables (IVs) for testing the multiple correlation and $n>104+m$ for testing IVs. In our study there was five IVs and the calculated sample number was $50+8(5)=90$ cases and $104+5=109$ cases for testing IVs. These calculations were based on significance level of $5 \%(\alpha=0.05)$ and $80 \%$ power $(p=0.20)$ [20]. All statistical tests were two-sided and a $\mathrm{p}<0.05$ was recognized as the statistical significance level.

\section{Results}

\section{Characteristics of the study subjects}

Anthropometric, clinical and biochemical characteristics of the study subjects are presented in Table 1 . Of the total 151 subjects [male: $85(56.3 \%)$ and female: $66(43.7 \%)]$, prediabetes groups such as IFG and IGT were $55(36.4 \%)$ and $96(63.6 \%)$ respectively. When they were stratified by liver ultrasonography according to the grades of NAFLD, there were $84(55.6 \%)$ in grade $0,53(35.1 \%)$ in grade $1,11(7.3 \%)$ in grade 2 and $3(2.0 \%)$ in grade 3 respectively. In NAFLD group, grade 1 , grade 2 and grade 3 subjects were $53(79.1 \%), 11(16.4 \%)$ and 3 $(4.5 \%)$, while, in the non NAFLD group the frequency was $84(100 \%)$ in grade 0 respectively. The obese NAFLD subjects were characterized by significantly highest levels of waist to hip ratio $(\mathrm{p}=0.026)$, systolic and diastolic blood pressure $(\mathrm{p}=0.004)$, total cholesterol $(\mathrm{p}=0.015)$, triglyceride $(\mathrm{p}=0.010)$, gamma-glutamyl transaminase $(\mathrm{p}=0.001)$, aspartate transaminase $(\mathrm{p}=0.024)$, fasting serum insulin $(\mathrm{p}=0.001)$, postprandial serum insulin $(\mathrm{p}<0.001)$, and fatty liver index $(\mathrm{p}<0.001)$, respectively as well as significantly lower levels of HOMA\%B ( $\mathrm{p}=0.005)$ and HOMA\%S ( $\mathrm{p}=0.025)$ compared to the non NAFLD subjects.

\begin{tabular}{|c|c|c|c|c|}
\hline \multirow{2}{*}{ Variables } & Total & Non NAFLD & NAFLD & \multirow{2}{*}{$p$ value } \\
\hline & $(n=151)$ & $(n=84)$ & $(n=67)$ & \\
\hline IFG & $55(36.4)$ & $33(39.3)$ & $22(32.8)$ & 0.593 \\
\hline IGT & $96(63.6)$ & $51(60.7)$ & $45(67.2)$ & \\
\hline Male, $n(\%)$ & $85(56.3)$ & $47(56.0)$ & $38(56.7)$ & 0.926 \\
\hline Female, $\mathrm{n}(\%)$ & $66(43.7)$ & $37(44.0)$ & $29(43.3)$ & \\
\hline Age (years) & $45.08 \pm 9.37$ & $44.11 \pm 9.35$ & $46.30 \pm 9.33$ & 0.154 \\
\hline BMI $\left(\mathrm{kg} / \mathrm{m}^{2}\right)$ & $25.95 \pm 4.42$ & $25.37 \pm 4.65$ & $26.66 \pm 4.04$ & 0.07 \\
\hline $\begin{array}{l}\text { Obese } \\
\text { prediabetes, } \\
\mathrm{n}(\%)\end{array}$ & $24(15.9)$ & $11(13.1)$ & $13(19.4)$ & 0.295 \\
\hline WC $(\mathrm{cm})$ & $89.96 \pm 8.21$ & $88.93 \pm 8.05$ & $91.25 \pm 8.28$ & 0.084 \\
\hline $\mathrm{HC}(\mathrm{cm})$ & $95.68 \pm 8.44$ & $95.35 \pm 8.73$ & $96.10 \pm 8.11$ & 0.582 \\
\hline WHR & $0.94 \pm 0.04$ & $0.93 \pm 0.04$ & $0.95 \pm 0.04$ & 0.026 \\
\hline Fat mass $(\%)$ & $29.58 \pm 7.58$ & $28.76 \pm 7.21$ & $30.62 \pm 7.96$ & 0.135 \\
\hline $\mathrm{SBP}(\mathrm{mmHg})$ & $122.69 \pm 24.58$ & $116.73 \pm 19.64$ & $130.16 \pm 28.03$ & 0.001 \\
\hline $\mathrm{DBP}(\mathrm{mmHg})$ & $82.57 \pm 21.43$ & $78.11 \pm 18.27$ & $88.16 \pm 23.82$ & 0.004 \\
\hline $\begin{array}{l}\text { Hypertensive } \\
\text { prediabetes, } \\
\text { n (\%) }\end{array}$ & $38(25.2)$ & $13(15.5)$ & $25(37.3)$ & 0.002 \\
\hline Grade 0 & $84(55.6)$ & $84(100)$ & - & $<0.001$ \\
\hline Grade 1 & $53(35.1)$ & - & $53(79.1)$ & \\
\hline Grade 2 & $11(7.3)$ & - & $11(16.4)$ & \\
\hline Grade 3 & $3(2.0)$ & - & $3(4.5)$ & \\
\hline $\begin{array}{l}\text { FSG } \\
(\mathrm{mmol} / \mathrm{L})\end{array}$ & $5.84 \pm 0.45$ & $5.87 \pm 0.49$ & $5.81 \pm 0.41$ & 0.414 \\
\hline $\begin{array}{l}\text { PPSG } \\
(\mathrm{mmol} / \mathrm{L})\end{array}$ & $8.12 \pm 1.50$ & $7.95 \pm 1.52$ & $8.33 \pm 1.47$ & 0.121 \\
\hline $\mathrm{HbA}_{1 \mathrm{c}}(\%)$ & $5.82 \pm 0.58$ & $5.82 \pm 0.64$ & $5.82 \pm 0.50$ & 0.994 \\
\hline $\mathrm{TC}(\mathrm{mg} / \mathrm{dL})$ & $194.36 \pm 40.60$ & $187.24 \pm 38.07$ & $203.30 \pm 42.18$ & 0.015 \\
\hline $\mathrm{TG}(\mathrm{mg} / \mathrm{dL})$ & $170.40 \pm 102.38$ & $151.33 \pm 69.78$ & $194.30 \pm 129.07$ & 0.01 \\
\hline $\begin{array}{l}\mathrm{HDL}-\mathrm{c} \\
(\mathrm{mg} / \mathrm{dL})\end{array}$ & $36.99 \pm 7.50$ & $38.02 \pm 7.44$ & $35.69 \pm 7.44$ & 0.057 \\
\hline LDL-c (mg/dL) & $184.27 \pm 43.38$ & $186.33 \pm 41.14$ & $182.87 \pm 46.48$ & 0.736 \\
\hline $\operatorname{ALT}(\mathrm{U} / \mathrm{L})$ & $34.13 \pm 16.20$ & $32.19 \pm 15.37$ & $36.57 \pm 16.99$ & 0.099 \\
\hline GGT (U/L) & $31.53 \pm 14.12$ & $28.27 \pm 13.53$ & $35.61 \pm 13.88$ & 0.001 \\
\hline AST (U/L) & $30.36 \pm 15.07$ & $27.90 \pm 11.38$ & $33.45 \pm 18.33$ & 0.024 \\
\hline
\end{tabular}




\begin{tabular}{|l|c|c|c|c|}
\hline ALP $(\mathrm{U} / \mathrm{L})$ & $101.90 \pm 26.47$ & $102.93 \pm 27.34$ & $100.31 \pm 25.49$ & 0.592 \\
\hline $\mathrm{FSI}(\mathrm{mIU} / \mathrm{mL})$ & $14.98 \pm 6.42$ & $13.50 \pm 4.05$ & $16.84 \pm 8.17$ & 0.001 \\
\hline $\begin{array}{l}\mathrm{PPSI}(\mathrm{mIU} / \mathrm{mL}) \\
\text { HOMA\%B }\end{array}$ & $111.11 \pm 37.02$ & $103.65 \pm 28.89$ & $120.47 \pm 43.65$ & 0.005 \\
\hline HOMA\%S & $59.55 \pm 30.60$ & $64.311 \pm 35.12$ & $53.38 \pm 22.62$ & 0.025 \\
\hline HOMA-IR & $1.98 \pm 0.80$ & $1.79 \pm 0.51$ & $2.21 \pm 1.01$ & 0.001 \\
\hline $\begin{array}{l}\text { NEFA } \\
(\mathrm{mmol} / \mathrm{L})\end{array}$ & $0.55 \pm 0.26$ & $0.50 \pm 0.26$ & $0.62 \pm 0.25$ & 0.01 \\
\hline FLI & $45.90 \pm 22.28$ & $39.85 \pm 21.14$ & $53.47 \pm 21.49$ & $<0.001$ \\
\hline
\end{tabular}

Data are expressed as means \pm SD or number of subjects (\%); the level of significance at $p<0.05$ calculated by Student's t test; $n=$ number of subjects. IFG: impaired fasting glucose; IGT: impaired glucose tolerance; NAFLD: nonalcoholic fatty liver disease.

Table 1: Anthropometric, clinical and biochemical characteristics among study participants based on the presence or absence of nonalcoholoic fatty liver disease.

\section{Serum NEFA and HOMA-IR levels in different grades of NAFLD}

Compared to the non NAFLD counterparts, NAFLD subjects showed significantly higher levels of serum NEFA ( $0.62 \pm 0.25$ vs. 0.50 $\pm 0.26 \mathrm{mmol} / \mathrm{L}, p=0.010)$ and HOMA-IR $(2.21 \pm 1.01$ vs. $1.79 \pm 0.51$, $p=0.001)$ resembling their gradual increase with progressing the severity of different grades of NAFLD (Table 1, Figures 1 and 2).

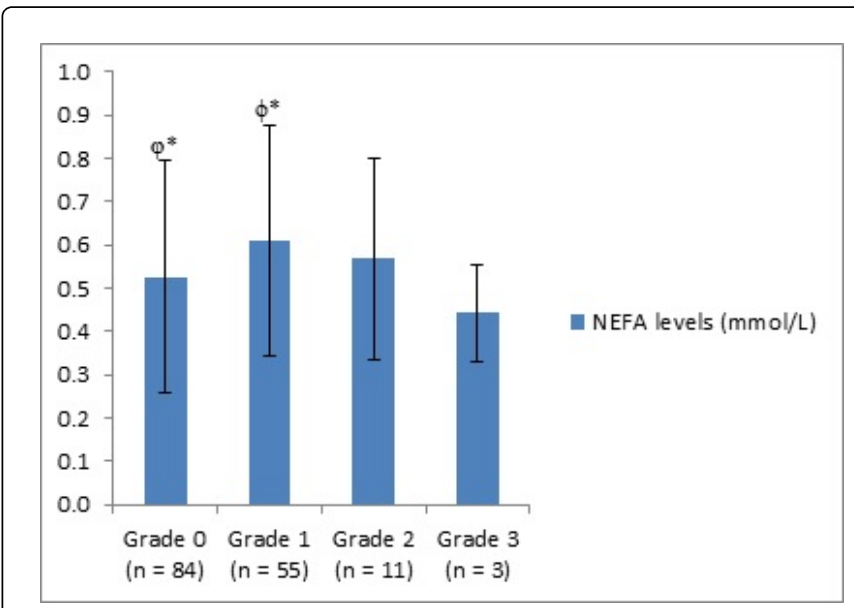

Figure 1: Serum NEFA levels based on NAFLD. Data are presented as mean $\pm \mathrm{SD}$; $\mathrm{n}=$ number of subjects; the level of significance at $p<0.05$ calculated by ANOVA. ${ }^{* * *} p<0.001,{ }^{* *} p<0.01$ and $\phi^{*} p<0.05$ considered statistically significant when compared with grade 0 NAFLD group; $\varphi^{* * *} p<0.001, \varphi^{* *} p<0.01$ and $\varphi^{*} p<0.05$ considered statistically significant when compared with grade 1 NAFLD group.

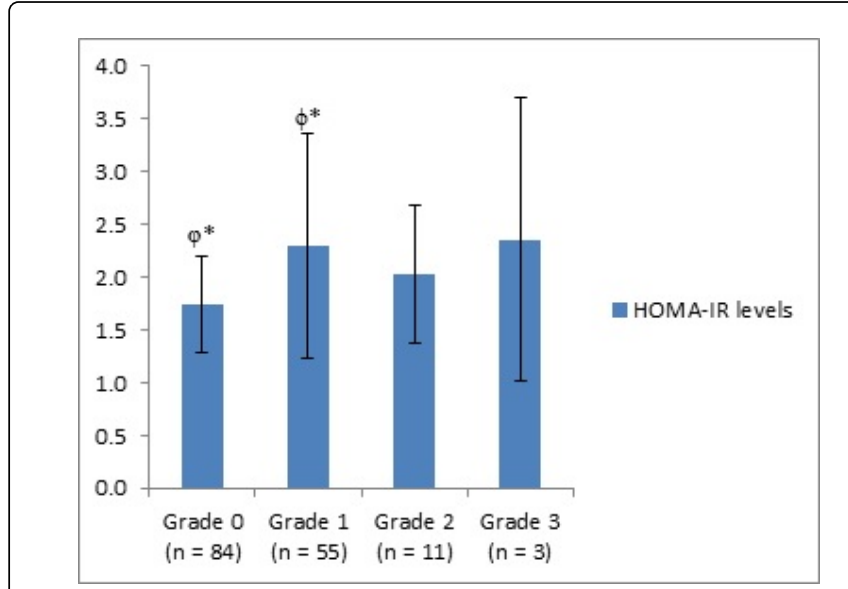

Figure 2: HOMA-IR levels based on NAFLD. Data are presented as mean $\pm \mathrm{SD}$; $\mathrm{n}=$ number of subjects; the level of significance at $p<0.05$ calculated by ANOVA. ${ }^{* * *} p<0.001,{ }^{* * *} p<0.01$ and $\phi^{*} p<0.05$ considered statistically significant when compared with grade 0 NAFLD group; $\varphi^{* * *} p<0.001, \varphi^{* *} p<0.01$ and $\varphi^{*} p<0.05$ considered statistically significant when compared with grade 1 NAFLD group.

\section{Association of serum NEFA and HOMA-IR with NAFLD subjects}

The association of serum NEFA and HOMA-IR with NAFLD subjects is shown in Table 2 using NAFLD group (NAFLD group as a dichotomous variable where non NAFLD subjects considered as reference) as the dependent variable and age, body mass index, triglyceride, HOMA-IR, and serum NEFA as independent variables. In this binary logistic regression analysis, serum NEFA (OR=6.18; $95 \%$ CI: 1.54 to $24.84, p=0.010)$ and HOMA-IR (OR=1.92; 95\% CI: 1.09 to $3.40, p=0.024)$ were found to be significant determinants of NAFLD after adjusting the effects of pertinent variables of age, body mass index, and triglyceride respectively.

\begin{tabular}{|c|c|c|c|c|c|c|}
\hline \multirow[b]{2}{*}{ Variables } & \multirow[b]{2}{*}{ Coefficient } & \multirow[b]{2}{*}{ SE } & \multirow[b]{2}{*}{$p$ value } & \multirow[b]{2}{*}{ OR } & \multicolumn{2}{|l|}{$95 \% \mathrm{Cl}$} \\
\hline & & & & & $\begin{array}{l}\text { Lower } \\
\text { Bound }\end{array}$ & $\begin{array}{l}\text { Upper } \\
\text { Bound }\end{array}$ \\
\hline Constant & -6.693 & 1.698 & 0 & 0.001 & - & - \\
\hline Age (years) & 0.041 & 0.02 & 0.038 & 1.042 & 1.002 & 1.083 \\
\hline BMI $\left(\mathrm{kg} / \mathrm{m}^{2}\right)$ & 0.055 & 0.043 & 0.194 & 1.057 & 0.972 & 1.149 \\
\hline $\mathrm{TG}(\mathrm{mg} / \mathrm{dL})$ & 0.005 & 0.003 & 0.059 & 1.005 & 1 & 1.011 \\
\hline HOMA-IR & 0.656 & 0.29 & 0.024 & 1.928 & 1.092 & 3.404 \\
\hline NEFA (mmol/L) & 1.822 & 0.71 & 0.01 & 6.185 & 1.54 & 24.849 \\
\hline \multicolumn{7}{|c|}{$\begin{array}{l}\text { Dependent variable: NAFLD as dichotomous variable; Adjusted } \mathrm{R}^{2}=0.166 \text {; the } \\
\text { level of significance at } p<0.05 \text {; SE: standard error; CI: confidence interval; } \\
\text { HOMA-IR: insulin resistance assessed by homeostasis model assessment; } \\
\text { NAFLD: nonalcoholic fatty liver disease. }\end{array}$} \\
\hline
\end{tabular}

Table 2: Binary logistic regression analysis taking NAFLD group (NAFLD vs. non NAFLD considered as reference) as dependant variable after adjusting the effects of major confounders. 


\begin{tabular}{|l|c|c|c|c|c|}
\hline \multirow{2}{*}{ Variables } & \multirow{2}{*}{$\beta$ value } & \multirow{2}{*}{$\boldsymbol{t}$ value } & \multirow{2}{*}{$\boldsymbol{p}$ value } & \multicolumn{2}{c|}{$95 \% \mathrm{Cl}$} \\
\cline { 5 - 6 } & & & & $\begin{array}{c}\text { Lower } \\
\text { Bound }\end{array}$ & $\begin{array}{c}\text { Upper } \\
\text { Bound }\end{array}$ \\
\hline Constant & - & 2.574 & 0.013 & 28.79 & 229.666 \\
\hline BMI $\left(\mathrm{kg} / \mathrm{m}^{2}\right)$ & -0.348 & -3.154 & 0.003 & -3.222 & -0.721 \\
\hline FSG $(\mathrm{mmol} / \mathrm{L})$ & -0.188 & -1.597 & 0.116 & -23.603 & 2.648 \\
\hline PPSG $(\mathrm{mmol} / \mathrm{L})$ & -0.215 & -1.863 & 0.067 & -6.939 & 0.247 \\
\hline HbA $1 \mathrm{1c}(\%)$ & 0.357 & 3.101 & 0.003 & 5.726 & 26.533 \\
\hline PSI (mIU/mL) & -0.367 & -3.305 & 0.002 & -0.339 & -0.083 \\
\hline NEFA $(\mathrm{mmol} / \mathrm{L})$ & -0.195 & -1.849 & 0.049 & -36.514 & 1.432 \\
\hline
\end{tabular}

Dependent variable: $\mathrm{HOMA} \% \mathrm{~S}$, insulin sensitivity assessed by homeostasis model assessment; Adjusted $\mathrm{R}^{2}=0.353$; the level of significance at $p<0.05 ; \beta$ : regression coefficient; $\mathrm{Cl}$ : confidence interval; NAFLD: nonalcoholic fatty liver disease.

Table 3: Multiple linear regression analysis to see the association of nonesterified fatty acid with insulin sensitivity after adjusting the effects of major confounders.

The results of multiple linear regression analysis are demonstrated in Table 3 using HOMA\%S as the dependent variable and body mass index, glycemic profile, postprandial serum insulin, and serum NEFA as independent variables. HOMA\%S showed significant negative association with serum NEFA ( $\beta=-0.195, p=0.049)$ after adjusting the effects of major confounding variables of body mass index, glycemic profile, and postprandial serum insulin respectively.

\section{Discussion}

The investigation of biomarkers responsible for the presence of NAFLD among prediabetic individuals is important because number risk factors are involved in the progression of prediabetes to diabetes. Insulin resistance which is the predominant feature of NAFLD as well as in prediabetes is also the concomitant risk factor of liver related morbidity and mortality. Different treatment strategies of NAFLD based on glycemic and lipid control, however, the particular origin from where the accumulation of TAG has occurred within the hepatocytes should have greater attention on the treatment regimen or new targets for therapy. In the present study, we used a noninvasive ultrasound method for the detection of NAFLD because of its accuracy and readily available tool in diagnosing NAFLD. Shannon et al. [21] found a significant positive correlation between USG and liver biopsy with a Spearman's coefficient of 0.80 [CI $(0.71,0.88)$, p value $<0.001]$.

NAFLD subjects in the Bangladeshi population have previously been shown to have insulin resistance; however, the underlying mechanism(s) of this defect is still unclear. In our settings, we found significantly higher levels of NEFA in NAFLD subjects compared to the controls. This observation is in agreement with a number of studies $[22,23]$. Donnelly et al. [24] found a higher TAG content in the liver arises from the NEFA pool in both fasting and fed state which is the major risk factor of NAFLD. NEFA levels also simultaneously increased with increasing the severity of NAFLD.

Our study results showed a significantly higher level of fasting and postprandial serum insulin in NAFLD subjects compared to non NAFLD subjects. In accordance with Lass et al. [25] revealed that impaired insulin action failed to inhibit the lipolysis of stored TAG in adipose tissue during fasting state, which then activates the hormonesensitive lipase action catalyzing the hydrolytic release of fatty acids from the TAG moiety and increases the influx of plasma NEFA pool. Hence, the newly made fatty acid arises in the hepatocytes not only from glycerol moiety by DNL in the fasting state but also from dietary fats in the fed state where the fats are mobilized as chylomicron remnants and secreted as lipoprotein TAG. Donnelly et al. [24,25] revealed DNL accounted for $26 \%$ of liver TAG and 23\% VLDL-TAG content in hyperinsulinemic subjects with NAFLD. Hyperinsulinemia and hyperglycemia may also activate some transcriptional factors that stimulate gene expression of most enzymes in humans thereby activating lipogenesis in hepatocytes. This is in line with the study of Sanyal et al. [23] where elevated insulin concentrations failed to suppress adipose tissue lipolysis, a result that suggested a definite source of fatty acids that has an important role in the development of NAFLD.

After adjustment for significant predictors (age, body mass index, and triglyceride), our study results revealed a strong association between serum NEFA and HOMA-IR with NAFLD group. In agreement with Farell et al. [26] reported a positive correlation of abdominal obesity with the prevalence of NAFLD, or NASH respectively, and is closely related to insulin resistance, a major pathogenetic factor for developing NASH. A cohort study conducted by Milić et al. [27] indicating the obesity indices such as high body mass index or waist circumference as a key risk factor for NAFLD. In the study of Leite et al. [28], central obesity is an independent risk factor for NAFLD development. With increasing the central obesity, insulin resistance increases the lipolysis of adipose tissue TAG thereby releasing NEFA pool. Portal entry of NEFA from adipose fat depot as well as the dietary intake increases the levels of the TAG by reesterification with the glycerol moiety or DNL in the hepatocytes. Increased production of TAG in both fasting and fed state due to impaired insulin action then accumulated within the hepatocytes and is a leading cause of NAFLD. In contrast, Diraison et al. [22] reported that the uptake of plasma NEFAs by the liver is not responsible for the development of NAFLD because IR reduces the glucose uptake and glyceroneogenesis in adipose tissue which in turn lowers the esterification of glycerol moiety as well as TAG synthesis.

In our settings, increased serum NEFA which is considered as a crucial biomarker of NAFLD showed significant positive association with reduced insulin sensitivity (HOMA\%S) after adjusting the effects of pertinent confounders (body mass index, glycemic profile, and postprandial serum insulin respectively). Elevated levels of plasma NEFA arises during fasting state mainly from hydrolysis of the TAG by lipase action within the adipocyte, however, after a fatty meal which is another important route of generation of plasma NEFA also enhanced by postprandial insulin by increasing the lipase action of dietary fats. In the adipose tissue, hormone sensitive lipoprotein lipase catalyzes the hydrolysis of stored TAG and released fatty acids that undergo $\beta$ oxidation for the production of ATP according to the demand of cells. However, when energy is available the excess fatty acid reenters the adipose tissue and stored as a TAG, but a proportion always escapes and joins the plasma NEFA pool [8]. In healthy individuals during the fasting state, the increased production of the TAG from the NEFA pool in hepatocytes is very low, however, it is rapidly increased following meals by DNL from the circulating lipogenesis precursors.

The molecular mechanism of lipogenesis in hepatocytes is still unclear. Insulin activates the membrane bound transcription factor 
Page 6 of 7

sterol regulatory element binding protein-1c (SREBP-1c), which then activates the transcription of genes coded for lipogenesis. During NAFLD, IR regulates the transcription of SREBP-1c thereby increasing the DNL, which also upregulated by insulin and is likely to be activated by hyperinsulinemia during postprandial state. Lipogenesis is also regulated by glucose precursors by activating the carbohydrate response element binding protein (ChREBP), which induces the transcription of pyruvate kinase gene and provides the precursors for lipogenesis [29]. ChREBP also stimulates gene expression of most enzymes involved in lipogenesis [30]. Thus, insulin resistance in adipose tissue plays a crucial role as a pathogenic factor of NAFLD.

Our study has several limitations. First, the diagnosis of NAFLD was confirmed by upper abdomen ultrasonography although liver biopsy is currently considered the gold standard method for the detection of hepatic steatosis. Second, correlation of adiposity markers with histologic pictures of different grades of NAFLD cannot be generalized. Third, cohorts of prediabetes with different subgroup having NAFLD are needed to re-formulate the association of serum NEFA with HOMA-IR among NAFLD individuals.

\section{Conclusion}

We found a significantly higher level of serum NEFA among the prediabetic individuals having NAFLD which also showed a gradual increase by growing the severity of NAFLD. Insulin resistance is one of the basic defects in the prediabetic state has also been considered a detrimental risk factor in the elevation of serum NEFAs leading to the development of NAFLD. The data also indicate that increased level of serum NEFA is a pathophysiological determinant of NAFLD in Bangladeshi prediabetic subjects and it seems that the elevation is mediated by reduced insulin sensitivity during this disorder. Future studies with large prospective design are warranted to explore the association of serum NEFA with different subgroups of prediabetes along with different grades of NAFLD.

\section{Author Contributions Statement}

IAH performed the data collection, precision of lab test analysis, statistical analysis of data, manuscript draft and conducted the whole research MRS analyzed and interpreted the data; LA conceived and designed the experiments, critical review of the manuscript and facilitat-ed reagents/materials/analysis tools. All of the authors read approved the final manuscript.

\section{Acknowledgement}

We express our sincerest gratitude to the study participants, laboratory personnel and staff of the BIHS General Hospital for their immense cooperation during the entire study. This research was partially supported by grants from the Bangladesh Medical Research Council and Government of the People's Republic of Bangladesh, Ministry of Science and Technology (No.39.00.0000.012.002.005.16-375).

\section{Declaration of Conflicting Interests}

The authors declare that they have no conflict of interest associated with this paper.

\section{Funding}

This study was partially supported by grants from the Bangladesh Medical Research Council and Govt. of the People's Republic of Bangladesh, Ministry of Science and Technology (No. 39.012.002.02.01.016.2013-335).

\section{References}

1. Bellentani S, Saccoccio G, Masutti F, Crocè LS, Brandi G, et al. (2000) Prevalence of and risk factors for hepatic steatosis in northern Italy. Ann Intern Med 132: 112-117.

2. Rinella ME (2015) Nonalcoholic fatty liver disease: a systematic review. JAMA 313: 2263-2273.

3. Gaggini M, Morelli M, Buzzigoli E, DeFronzo RA, Bugianesi E, et al. (2013) Non-alcoholic fatty liver disease (NAFLD) and its connection with insulin resistance, dyslipidemia, atherosclerosis and coronary heart disease. Nutrients 5: 1544-1560.

4. Karpe F, Dickmann JR, Frayn KN (2011) Fatty Acids, Obesity, and Insulin Resistance: Time for a Reevaluation. Diabetes 60: 2441-2449.

5. Nielsen S, Guo Z, Johnson CM, Hensrud DD, Jensen MD (2004) Splanchnic lipolysis in human obesity. J Clin Invest 113: 1582-1588.

6. Finucane FM, Sharp SJ, Hatunic M, Sleigh A, De Lucia Rolfe E, et al. (2013) Intrahepatic Lipid Content and Insulin Resistance Are More Strongly Associated with Impaired NEFA Suppression after Oral Glucose Loading Than with Fasting NEFA Levels in Healthy Older Individuals. Int J Endocrinol 2013: 7.

7. Nagle CA, An J, Shiota M, Torres TP, Cline GW, et al. (2007) Hepatic overexpression of glycerol-sn-3-phosphate acyltransferase 1 in rats causes insulin resistance. J Biol Chem 282: 14807-14815.

8. Evans K, Burdge GC, Wootton SA, Clark ML, Frayn KN (2002) Regulation of dietary fatty acid entrapment in subcutaneous adipose tissue and skeletal muscle. Diabetes 51: 2684-2690.

9. McQuaid SE, Hodson L, Neville MJ, Dennis AL, Cheeseman J, et al. (2011) Down regulation of adipose tissue fatty acid trafficking in obesity: a driver for ectopic fat deposition? Diabetes 60: 47-55.

10. Alam S, Fahim SM, Chowdhury MAB, Hassan MZ, et al. (2018) Prevalence and risk factors of non-alcoholic fatty liver diseasein Bangladesh. JGHOpen 2: 239-246.

11. Hossain IA, Akter S, Bhuiyan FR, Shah MR, Rahman MK, et al. (2016) Subclinical inflammation in relation to insulin resistance in prediabetic subjects with nonalcoholic fatty liver disease. BMC Res Notes 9: 266.

12. World Health Organization (2006) Definition and diagnosis of diabetes mellitus and intermediate hyperglycaemia. Report of a WHO/IDF consultation. Geneva: World Health Organization.

13. WHO Expert Consultation (2004) Appropriate body-mass index for Asian populations and its implications for policy and intervention strategies. Lancet 363: 157-163.

14. Whitworth JA, Chalmers J (2004) World health organizationinternational society of hypertension (WHO/ISH) hypertension guidelines. Clin Exp Hypertens 26: 747-752.

15. World Health Organization (1999) Definition and diagnosis and classification of diabetes mellitus and its complications: report of a WHO consultation. Part 1. Geneva, Switzerland: WHO pp: 32-33.

16. Friedewald WT, Levy RI, Fredrickson DS (1972) Estimation of the concentration of low density lipoprotein cholesterol in plasma without use of the preparative ultracentrifuge. Clin Chem; 18: 499-502.

17. Matthews DR, Hosker JP, Rudenski AS, Naylor BA, Treacher DF, et al. (1985) Homeostasis model assessment: insulin resistance and beta-cell function from fasting plasma glucose and insulin concentrations in man. Diabetologia 28: 412-419.

18. Lee SS, Park SH, Kim HJ, Kim SY, Kim MY, et al. (2010) Non-invasive assessment of hepatic steatosis: prospective comparison of the accuracy of imaging examinations. J Hepatol 52: 579-585. 
Citation: Hossain IA, Shah MR, Ali L (2018) Association of Serum Nonesterified Fatty Acid and Insulin Resistance with Nonalcoholic

Page 7 of 7

19. Bedogni G, Bellentani S, Miglioli L, Masutti F, Passalacqua M, et al (2006) The Fatty Liver Index: a simple and accurate predictor of hepatic steatosis in the general population. BMC Gastroenterol 6: 33.

20. Green SB (1991) How Many subjects does it take to do a regression analysis? Multivariate Behav Res 26: 499-510.

21. Shannon A, Alkhouri N, Carter-Kent C, Monti L, Devito R, et al. (2011) Ultrasonographic Quantitative Estimation of Hepatic Steatosis in Children with Nonalcoholic Fatty Liver Disease. J Pediatr Gastroenterol Nutr 53: 190-195.

22. Diraison F, Moulin P, Beylot M (2003) Contribution of hepatic de novo lipogenesis and reesterification of plasma nonesterified fatty acid to plasma triglyceride synthesis during non-alcoholic fatty liver disease. Diab Metab 29: 478-485.

23. Sanyal AJ, Campbell-Sargent C, Mirshahi F, Rizzo WB, Contos MJ, et al. (2001) Nonalcoholic steatohepatitis: association of insulin resistance and mitochondrial abnormalities. Gastroenterology 120: 1183-1192.

24. Donnelly KL, Margosian MR, Sheth SS, Lusis AJ, Parks EJ (2004) Increased lipogenesis and fatty acid reesterification contribute to hepatic triacylglycerol stores in hyperlipidemic Txnip-/-mice. J Nutr 134 1475-1480.
25. Lass A, Zimmermann R, Oberer M, Zechner R (2010) Lipolysis-A highly regulated multi-enzyme complex mediates the catabolism of cellular fat stores. Prog Lipid Res 50: 14-27.

26. Farrell GC, Larter CZ (2006) Nonalcoholic fatty liver disease: From steatosis to cirrhosis. Hepatology 43: 99-112.

27. Milić S, Lulić D, Štimac D (2014) Non-alcoholic fatty liver disease and obesity: biochemical, metabolic and clinical presentations. World J Gastroenterol 20: 9330-9337.

28. Leite NC, Salles GF, Araujo AL, Villela-Nogueira CA, Cardoso CR (2009) Prevalence and associated factors of non-alcoholic fatty liver disease in patients with type-2 diabetes mellitus. Liver Int 29: 113-119.

29. Yamashita H, Takenoshita M, Sakurai M, Bruick RK, Henzel WJ, et al. (2001) A glucose-responsive transcription factor that regulates carbohydrate metabolism in the liver. Proc Natl Acad Sci USA 98: 9116-9121.

30. Iizuka K, Bruick RK, Liang G, Horton JD, Uyeda K (2004) Deficiency of carbohydrate response element binding protein (ChREBP) reduces lipogenesis as well as glycolysis. Proc Natl Acad Sci USA 101: 7281-7286. 